\title{
Correction to: Disc inflammation and Modic changes show an interaction effect on recovery after surgery for lumbar disc herniation
}

\author{
Niek Djuric ${ }^{1}$ - Xiaoyu Yang ${ }^{1} \cdot$ Raymond W. J. G. Ostelo $^{2,3} \cdot$ Sjoerd G. van Duinen ${ }^{4} \cdot$ Geert J. Lycklama à Nijeholt $^{5}$. \\ Bas F. W. van der Kallen ${ }^{4}$. Wilco C. Peul ${ }^{1,5}$. Carmen L. A. Vleggeert-Lankamp ${ }^{1}$
}

Published online: 31 December 2019

(c) Springer-Verlag GmbH Germany, part of Springer Nature 2019

\section{Correction to: European Spine Journal (2019) 28:2579-2587 https://doi.org/10.1007/s00586-019-06108-9}

In Tables 3 and 4: In the first column and row, the text reads "Mixed model test (patients with Modic changes)". This should have been just "Mixed model". The complete correct Tables 3 and 4 are given below.

The original article can be found online at https://doi.org/10.1007/ s00586-019-06108-9.

Niek Djuric

n.djuric@lumc.nl

1 Department of Neurosurgery, Leiden University Medical Center, Albinusdreef 2, 2300 RC Leiden, The Netherlands

2 Department of Epidemiology, VU Medical Centre, Amsterdam, The Netherlands

3 Department of Health Sciences, Faculty of Science, Amsterdam Movement Sciences Research Institute, Vrije Universiteit, Amsterdam, The Netherlands

4 Department of Pathology, Leiden University Medical Center, Leiden, The Netherlands

5 Haaglanden Medical Centre, The Hague, The Netherlands
Table 3 Baseline main and interaction effects of MC2 and inflammation on clinical outcomes

\begin{tabular}{llll}
\hline Mixed model & $\begin{array}{l}\text { RDQ score } \\
F \text { value } \\
(p \text { value })\end{array}$ & $\begin{array}{l}\text { VAS leg score } \\
F \text { value } \\
(p \text { value })\end{array}$ & $\begin{array}{l}\text { VAS back score } \\
F \text { value } \\
(p \text { value })\end{array}$ \\
\hline MC2 effect & $0.01(p=1.000)$ & $2.61(p=1.000)$ & $0.78(p=1.000)$ \\
$\begin{array}{l}\text { Inflammation } \\
\text { effect }\end{array}$ & $0.34(p=1.000)$ & $1.25(p=1.000)$ & $0.40(p=1.000)$ \\
$\begin{array}{c}\text { Interaction } \\
\text { effect MC2* } \\
\text { inflammation }\end{array}$ & $0.07(p=1.000)$ & $\begin{array}{l}0.415 \\
(p=1.000)\end{array}$ & $3.45(p=0.315)$ \\
\hline
\end{tabular}

Two-way ANOVA was used, values are $F$ values with $p$ values, $n$ total $=119$. $p$ values are corrected for multiple testing according to Bonferroni ( 9 tests)

Table 4 Follow-up main and interaction effects of MC2 and inflammation on clinical outcomes

\begin{tabular}{llll}
\hline Mixed model & $\begin{array}{l}\text { RDQ score } \\
F \text { value } \\
(p \text { value })\end{array}$ & $\begin{array}{l}\text { VAS leg score } \\
F \text { value } \\
(p \text { value })\end{array}$ & $\begin{array}{l}\text { VAS back score } \\
F \text { value } \\
(p \text { value })\end{array}$ \\
\hline MC2 effect & $2.47(p=0.963)$ & $0.02(p=1.000)$ & $1.52(p=1.000)$ \\
$\begin{array}{l}\text { Inflammation } \\
\text { effect }\end{array}$ & $2.25(p=1.000)$ & $6.97(p=0.009)$ & $2.32(p=0.891)$ \\
$\begin{array}{c}\text { Interaction } \\
\text { effect MC2* }\end{array}$ & $7.10(p=0.009)$ & $4.69(p=0.090)$ & $0.75(p=1.000)$ \\
inflammation & & & \\
\hline
\end{tabular}

Mixed model analysis was used, values are $F$ values with $p$ values, $n$ total $=96$. $p$ values are corrected for multiple testing according to Bonferroni ( 9 tests)

Publisher's Note Springer Nature remains neutral with regard to jurisdictional claims in published maps and institutional affiliations. 